\title{
RASPBERRY PI BASED LIQUID FLOW MONITORING AND CONTROL
}

\author{
N.Suresh ${ }^{1}$, E.Balaji ${ }^{2}$, K.Jeffry Anto ${ }^{3}$, J.Jenith ${ }^{4}$ \\ ${ }^{l}$ Assistant Professor, Dept of EIE, Sathyabama University, Tamilnadu, India \\ ${ }^{2}$ Student, Dept of EIE, Sathyabama University, Tamil Nadu, India \\ ${ }^{3}$ Student, Dept of EIE, Sathyabama University, Tamil Nadu, India \\ ${ }^{4}$ Student, Dept of EIE, Sathyabama University, Tamil Nadu, India
}

\begin{abstract}
In industries there will be many big pipelines carrying various types of liquids, including the flammable liquids and corrosive liquids. It is necessary to control the flow rate of the liquid for enhancing the processes and to prevent the accidents. The paper proposes a methodology to monitor and control the liquid flow in the pipeline of industries through web server. There are many systems to do the same, but this is about to monitor and control the flow of liquid using Internet with the help of Raspberry pi and Arduino. The flow rate of the liquid is measured by Hall Effect sensor based flow meter. Arduino, a microcontroller development board reads the pulses from the flow meter and sends it to Raspberry pi, a microcomputer to control the electro valve which is connected to the pipeline. Server was setup by means of Raspberry pi..
\end{abstract}

Keywords: Arduino, Raspberry pi, Flow meter, Hall Effect sensor, electro valve.

\section{INTRODUCTION}

Measuring the flow of liquids is a critical need in many industrial plants. In some operations, the ability to conduct accurate flow measurements is so important that it can make the difference between making a profit or taking a loss. In other cases, inaccurate flow measurements or failure to take measurements can cause serious or even disastrous results. With most liquid flow measurement instruments, the flow rate is determined inferentially by measuring the liquid's velocity or the change in kinetic energy. Velocity depends on the pressure differential that is forcing the liquid through a pipe or conduit. Because the pipe's cross-sectional area is known and remains constant, the average velocity is an indication of the flow rate.

It is very important to know what a flow meter can do as well cannot do. Each type has both advantages and disadvantages. Technological improvements of flow meter must also be considered. It is always considered about the needs of the flow meter. The satisfaction received with the product depends on the care used in selecting and installing the device. Mistakes when installing the device is common. Every design has some tolerance to variable velocity conditions in the pipe. Without it accuracy and performance will not be correct. The biggest problem is with metering. The values may not be clearly understood that is they may be in different parameters. Regularly the meters have to be calibrated. All the flow meters require initial calibration. The recalibration depends on how well the meter fits the application. There are number of factors influence maintenance requirements and the life expectancy of flow meters. The major factor is matching the right instrument to the particular application. There are many other flow meters used in various places. They are monitored using the wired computer systems. The person can notice the flow only he is in the computer system and using the particular application to monitor the flow. So that for monitoring only the person needs an individual computer. The person cannot know the flow when he was not in the place of monitoring. The proposed system helps to monitor and control the flow of liquid through internet by mobile or computer.

\section{PROPOSED SYSTEM}

The proposed system to monitor the flow of liquid basically consists of electro-valve, flow meter, microcontroller microcomputer and web server. The block diagram of the system is shown in the fig. 1 . To measure the flow rate of the liquid, Hall Effect sensor based flow meter is used. Arduino and Raspberry $\mathrm{Pi}$ will act as a microcontroller and microcomputer respectively. The electro valve is physically connected to the pipeline in which the flow to be controlled. The electro valve is connected with the flow meter / flow sensor. The flow sensor measures the flow and generates analog pulse. The flow sensor/ flow meter is connected with arduino in order to read the pulses from the flow sensor. The arduino reads the analog pulse from the flow sensor and sends the signal to the raspberry pi which is the main component to control the electro valve. The Electro valve is electrically connected to the raspberry pi, by which is to be energized to open or close the valve. The raspberry pi is programmed to read the arduino signal and to control the electro valve. The raspberry $\mathrm{Pi}$ is also programmed to act as a Web server by which the electro valve is controlled using the LAN (Local Area Network), or internet connection. Arduino is responsible for collecting the data from the flow meter and sends it to the raspberry pi. Flow meter is interfaced with Arduino directly. 


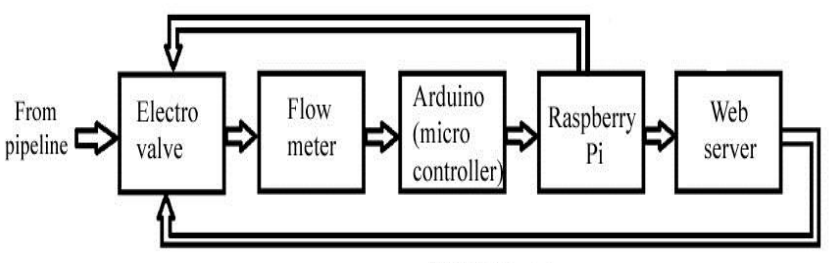

ON/OFF Control

Fig-1: Block diagram of the proposed system to control the flow of liquid.

The flow meter works on the principle of Hall Effect sensor. The Hall Effect sensor is a transducer whosw output voltage varies in response to a magnetic field. As the liquid flows through the flow meter, Hall Effect sensor senses the liquid flow and sends the corresponding data to Arduino through the interface. The next part involves the uploading of data that has been received from the arduino to raspberry pi. Raspberry $\mathrm{Pi}$ is a microcomputer which is of light weight that runs on Linux Operating System capable of handling various hardware interfaces. Arduino is directly connected to the Universal Serial Bus port that is available in raspberry pi. The python is used as a primary coding language by the raspberry pi to receive the data from the serial port and uploading it to the server and also running the server. The flow rate of the liquid can be controlled by electro valve activated by the raspberry pi through the internet. Raspberry pi is capable of controlling hardware using the GPIO pins (General Purpose Input/output pins) it has. The electro-valve is connected to the GPIO pins and is accessed through internet. The GPIO pins in raspberry pi are capable of supplying power. The electro-valve is a solenoid valve. This electro-valve will interrupt the liquid flow until $12 \mathrm{v}$ power supply is given. The power supply to the solenoid valve is given by means of GPIO pins from raspberry pi and controls it from the web server.

\section{HARDWARE}

The major components used in the proposed have been discussed briefly.

\subsection{Hall Effect Flow Sensor}

The flow rate of the liquid is measured with the help of Hall Effect sensor type flow meter. Hall Effect type flow sensor is shown in the fig.2.A Hall Effect sensor is a transducer that varies its output voltage in response to a magnetic field. The Hall Effect is the production of a voltage difference across an electrical conductor, transverse to an electric current in the conductor and a magnetic field perpendicular to the current Hall Effect sensors are used for proximity switching, positioning, speed detection, and current sensing applications. The flow meter is capable of measuring 1-30 Liters per minute. It can withstand the pressure of water less than or equal to 2.0 Mpa. The flow sensor is connected to a $1 / 2$ inch pipe. It senses the flow of liquid in the pipe and sends the corresponding analog signal to arduino.

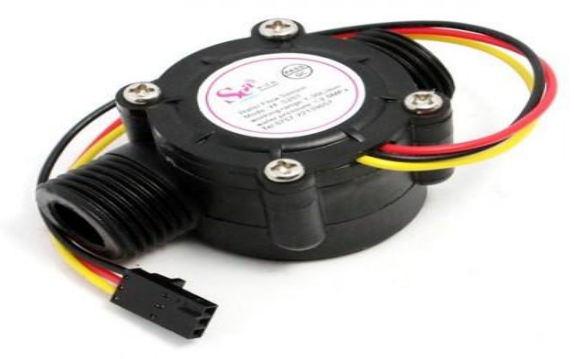

Fig-2: Flow meter sensor

\subsection{Solenoid Electro-valve}

A solenoid valve is an electromechanically operated valve. The valve is controlled by an electric current through a solenoid. Raspberry Pi controls the operation of the electrovalve. The picture of the electro-valve to control the flow of liquid is shown in the fig.3. The operating voltage of the electro-valve is $12 \mathrm{~V}$. The solenoid valve operates at a minimum of around $3 \mathrm{psi}$ and allows around $3 \mathrm{~L} / \mathrm{min}$ of flow, and it needs enough pressure to operate. It will interrupt the flow until $12 \mathrm{~V}$ is applied to the fast-on connectors on the solenoid.

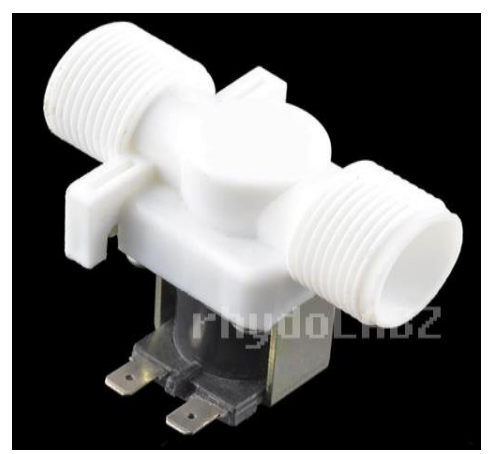

Fig-3: Solenoid Electro-valve

The electro valve is connected to the ON - OFF switches. The valve is connected to the electrical circuit which energizes the valve to open or close whenever necessary. When the OPEN switch is activated the valve gets de energized and lets the liquid to flow and when the close switch is pressed the valve gets energized by the electrical circuit and it gets closed and does not allow the liquid to flow.

\subsection{Arduino}

Arduino is a single board micro controller intended to make the application of interactive objects or environments more accessible. There are many types of Arduino available. The ARDUINO UNO REV3 model. has been used. It is shown in the fig.4. The Arduino Uno is a micro controller board based on the ATmega328. It has 14 digital input/output pins (of which 6 can be used as PWM outputs), 6 analog inputs, a $16 \mathrm{MHz}$ ceramic resonator, a USB connection, a power jack, 
an ICSP header, and a reset button. It contains everything needed to support the micro controller; simply connect it to a computer with a USB cable or power it with an AC-to-DC adapter or battery to get started.

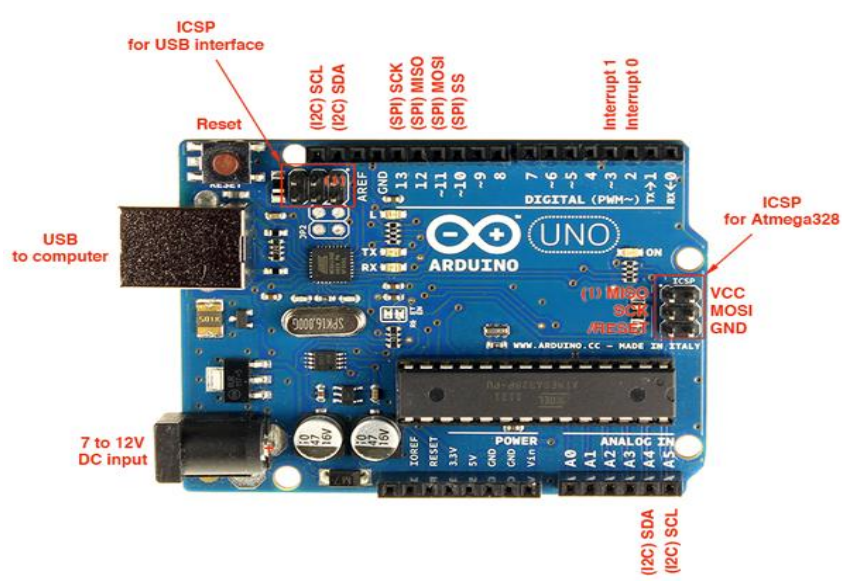

Fig-4: Arduino board

In order to program Arduino, arduino IDE (i.e.) arduino programming platform is installed in a Windows Pc. The program is written in the arduino ide and then uploaded to the arduino with the help of a serial cable Universal Serial Bus directly connected to the pc. The arduino will read the data from the flow meter and convert it as it is programmed. The data that is received from the flow meter in the form of analog data is converted to digital pulses by arduino so that raspberry pi can read it.

\subsection{Raspberry Pi}

Raspberry pi is a credit card sized single board computer which is capable of doing the entire job that an average desktop computer does Like spread sheets, word processing, Internet, Programming, Games etc .It consist of $512 \mathrm{mb}$ RAM, ARM v6 Processor,2 USB and an ethernet port, HDMI \& RCA ports for display,3.5mm Audio jack,SD card slot (bootable), General purpose I/O pins, runs on $5 \mathrm{v}$. The Raspberry Pi model is shown in fig.5.

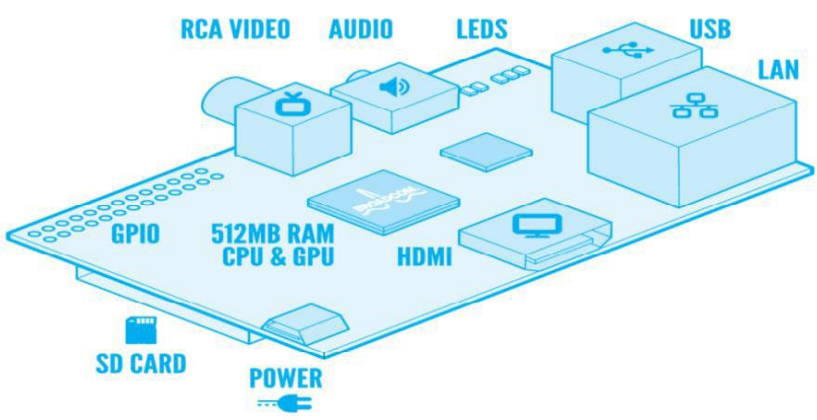

Fig.5: Rasperry Pi model

Raspberry pi runs on Linux kernel based operating systems. It boots and runs from the SD card. It does not have any internal memory other than the ROM. It has an SD card slot which is capable of reading up to $32 \mathrm{~GB}$. The GPIO pins of the raspberry pi are programmed using Python programming language. The Electro-valve is connected to GPIO pins with the help of transistor and relay so that we can switch on switch off it whenever needed.

\section{RESULTS AND DISCUSSION}

The prototype model to control the flow of liquid through server is shown in fig.6.Water is used as the liquid to test the setup.

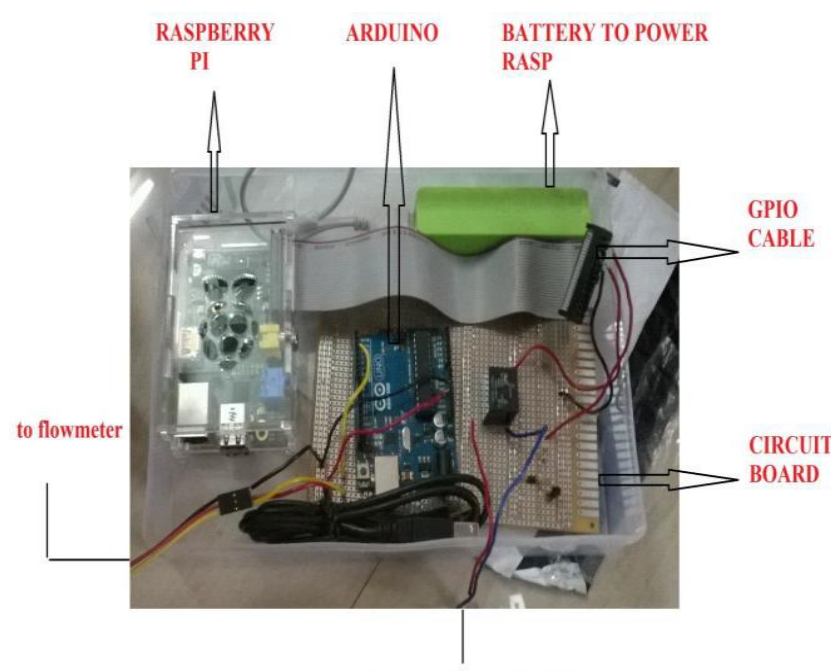

TO ELECTRO-VALVE

Fig-6: Prototype Model

Snapshot of the water flow setup is shown in fig.7. According to flow meter sensor's data sheet 5600 pulses $=1$ litres. The program is written to send the data every second. First of all, a small tank with low pressure was used. The data was like this (The liquid used here is water) 258 L/hour,321 L/hour,278 L/hour,345 L/hour etc. The water flow was very slow. But even when the water is turned OFF there were some reading like 20L/hour, 31L/hour, etc. indicating zero error. All the components were checked carefully, It was found to be loose connection between the interrupt pins in arduino, sending blank pulses to the serial port and has been rectified. Now the connections are checked again and the water flow is switched ON. Now there is no error and the data it shows is perfect when the water is switched OFF it correctly shows $0 \mathrm{~L} /$ hour

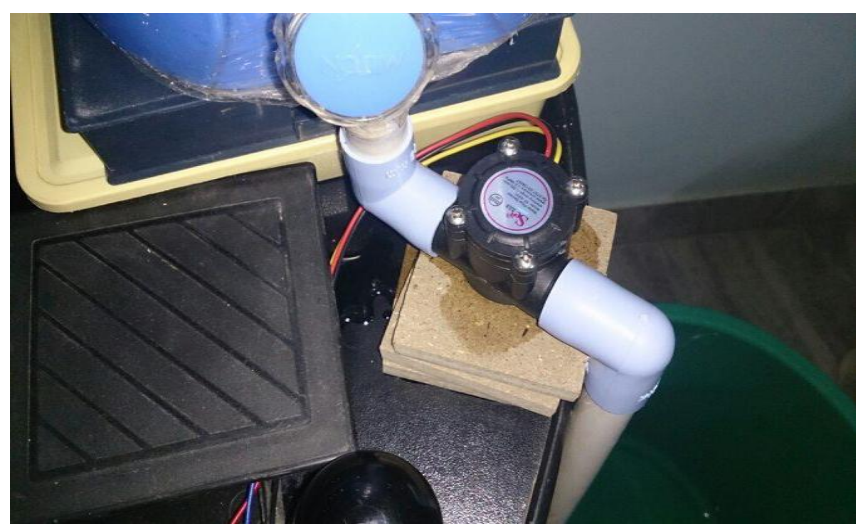

Fig-7: Snapshot of Water flow setup. 
The fig. 8 shows the monitoring of the liquid flow through the Smart phone. The electro-valve needs minimum of 1215 PSI to work. Initially, the electro-valve was connected to the small tank (low pressure), when triggered it does not open properly. Small tank that we used for flow meter did not suffice. So overhead tank has been used. After doing the plumbing connections, the electro-valve was triggered and it worked fine

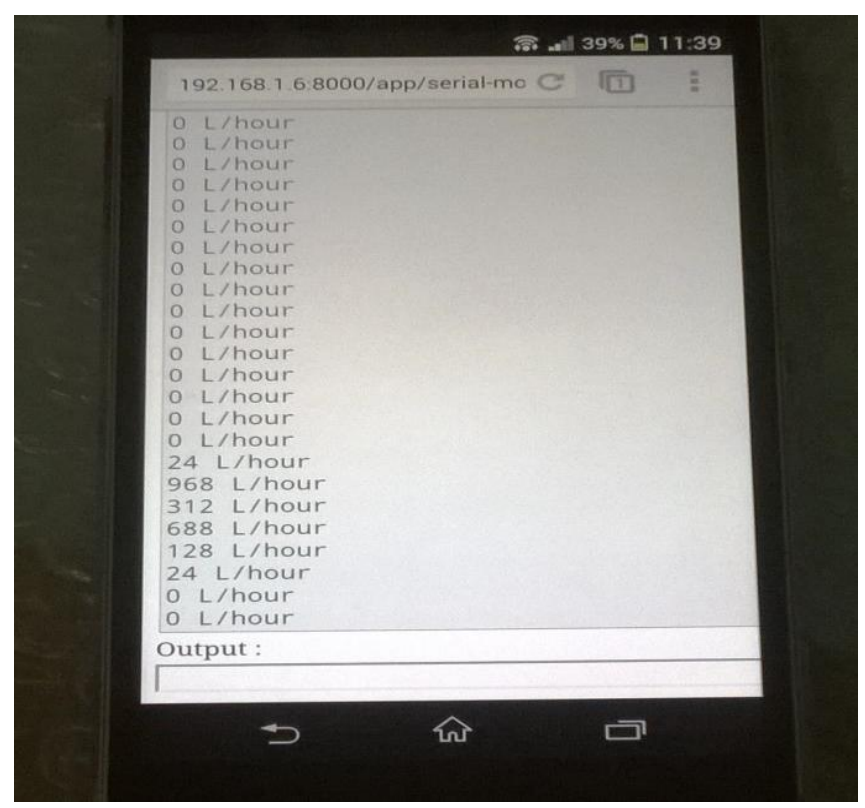

Fig-8: Liquid Flow Monitoring using Smart phone

In order to open the server in any browser the IP (internet protocol) address of the server need to be known.. The HTML page designed uses java script, so whatever the browser is used, it must be installed with java plugin or else the data will not be displayed properly. For security reasons we have assigned username and password for the user to monitor the data, to enter the website. When the web page opens, there will be two options Flow data and Control. The required option shall be selected

\section{CONCLUSION}

The Liquid flow can be monitored and controlled from anywhere in the world using internet through personal computer or Smartphone. The system has been tested for water as a flowing liquid successfully. The work can be extended to liquids that are used in various industries with careful considerations of parameters like pressure, temperature, corrosion, etc with appropriate use of flow sensors and electro-valve.

\section{REFERENCES}

[1]. Design of networking liquid flow measurement and management control system based on .NET, Ya-Ping Shi et al, IEEE Conference Publications, 2011.

[2]. Remote Measuring of Flow Meters for Petroleum Engineering and Other Industrial Applications , Abdelgawad, A. et al, IEEE Conference Publications, 2007.
[3]. A Hall Effect sensor based syringe injection rate detector , Mukherjee, $\mathrm{B}$ et al, IEEE Conference Publications, 2012

[4]. Data acquisition and control using Arduino-Android platform: Smart plug , Shajahan, A.H et al, IEEE Conference Publications, 2013

[5]. An arduino based system provided with GPS/GPRS shield for real time monitoring of traffic flows, Costanzo, A. IEEE Conference Publications,2013

[6]. Control and monitoring system of small water and wind power plant, Kovalcik, M. et al, IEEE Conference Publications, 2013.

[7]. Bluetooth communication using a touchscreen interface with the Raspberry Pi , Sundaram, G.S et al, IEEE Conference Publications, 2013.

[8]. Raspberry Pi based interactive home automation system through E-mail, Jain, S. et al, IEEE Conference Publications, 2014

[9]. Automated electric meter reading and monitoring system using ZigBee-integrated raspberry Pi single board computer via Modbus, Bonganay, A.C.D. et al, IEEE Conference Publications, 2014.

[10]. Precise dosage system for controlled liquid delivery based on fast MEMS based flow sensor, Goetz, M. et al, IEEE Conference Publications, 2009.

[11]. www.raspberrypi.org

[12]. www.arduino.cc 